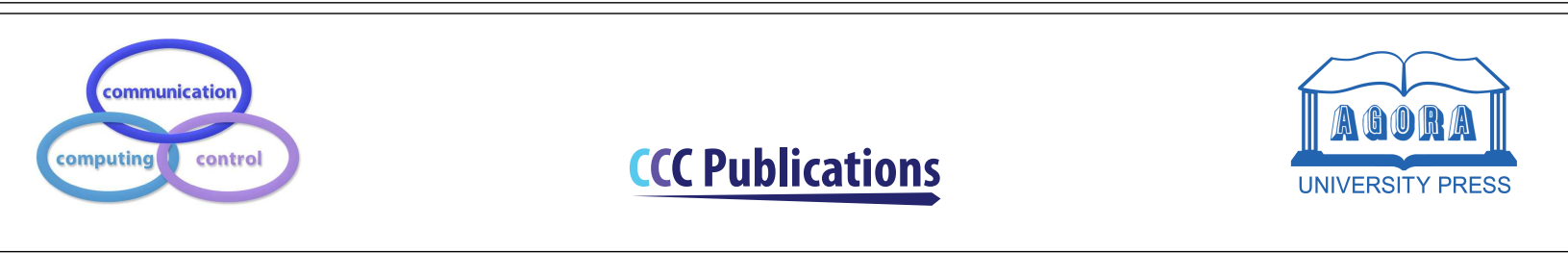

\title{
Dynamic Expert System-Based Geographically Adapted Malware Risk Evaluation Method
}

\author{
D. Vitkus, J. Jezukeviciute, N. Goranin
}

\author{
Donatas Vitkus* \\ Vilnius Gediminas Technical University, \\ Vilnius, Lithuania \\ *Corresponding author: d.vitkus@vgtu.lt

\section{Justina Jezukeviciute} \\ Vilnius Gediminas Technical University, \\ Vilnius, Lithuania \\ justina.jezukeviciute@stud.vgtu.lt \\ Nikolaj Goranin \\ Vilnius Gediminas Technical University, \\ Vilnius, Lithuania \\ nikolaj.goranin@vgtu.lt
}

\begin{abstract}
Fast development of information systems and technologies while providing new opportunities for people and organizations also make them more vulnerable at the same time. Information security risk assessment helps to identify weak points and preparing mitigation actions. The analysis of expert systems has shown that rule-based expert systems are universal, and because of that can be considered as a proper solution for the task of risk assessment automation. But to assess information security risks quickly and accurately, it is necessary to process a large amount of data about newly discovered vulnerabilities or threats, to reflect regional and industry specific information, making the traditional approach of knowledge base formation for expert system problematic. This work presents a novel method for an automated expert systems knowledge base formation based on the integration of data on regional malware distribution from Cyberthreat real-time map providing current information on newly discovered threats. In our work we collect the necessary information from the web sites in an automated way, that can be later used in a relevant risk calculation. This paper presents method implementation, which includes not only knowledge base formation but also the development of the prototype of an expert system. It was created using the JESS expert system shell. Information security risk evaluation was performed according to OWASP risk assessment methodology, taking into account the location of the organization and prevalent malware in that area.
\end{abstract}

Keywords: information security risk analysis, expert systems, knowledge base formation, JESS, information acquisition. 


\section{Introduction}

Information security risk assessment is a good way to manage the information security of the enterprise [1]. It helps not only in identifying weak points of enterprise information security management system but also in reducing costs of implementing information security controls because there is no necessity to implement all possible controls but only those, which are identified as necessary during the information security risk analysis.

Today's fast development of information systems and technologies provides new opportunities for people and organizations [5]. While it enables business to be faster and more competitive, it also makes them more vulnerable at the same time. Ontime conducted information security risk assessment allows to prepare a mitigation plan and reduce risks to an acceptable level [10]. On the other hand, companies need to employ information security risk manager or buy information security risk assessment service. Sometimes it becomes a problem, especially for small-medium enterprises, that is why automation of risk analysis process by employing expert systems is considered as a perspective approach.

This paper proposes a novel approach of knowledge base formation for expert systems compared to the traditional one, that requires real experts, knowledge base formation process is long, sometimes expensive [7]. It is suitable in cases where long-lasting data can be used, but not in information security risk assessment. Knowledge base used in information security risk assessment should reflect constantly changing threat landscape, evaluate the risk probability depending on the region and other factors.

The method proposed is based on the acquisition of data from trusted and open websites providing current information on newly discovered threats. The proposed method was implemented by data acquisition from the Cyberthreat real-time map website by Kaspersky Lab [19].

Also, a prototype of an expert system using a knowledge base with automatically acquired data was developed. The prototype was designed to perform the information security risk assessment according to the OWASP methodology [6]. The formed knowledge base was implemented on the Java Expert System Shell (JESS) [21]. The acquired data was automatically imported into JESS using XML format. It is necessary to state, that automatically acquired data has formed only part of a knowledge base, i.e. was integrated into the existing knowledge base and was used to estimate the malware probability, used in risk calculation in the specific region.

The paper is organized as follows. Section 1, current section, is an introduction. Section 2 presents related works in information security risk analysis and the development of expert systems knowledge base. Section 3 presents an approach of the integration of data on regional malware distribution from Cyber-threat real-time map into the knowledge base of risk analysis expert system for geographically adapted risk evaluation. Section 4 is results and discussions and finally, section 5 concludes the paper.

\section{Related work}

The analysis of expert systems has shown that rule-based expert systems are universal, and because of that, can be considered as a proper solution for the task of risk assessment automation. Expert systems can be a proper solution for enterprises because it allows to reduce costs for the risk management [2]. But to assess information security risks quickly and accurately, it is necessary to process a large amount of data about newly discovered vulnerabilities or threats, to reflect regional and industry specific information [9].

One of the most difficult points in creating expert systems is the creation of a knowledge base. In this part of the expert system are stored system rules, patterns, facts, data specifications and other structures [6]. It allows the inference engine to give the right answer to the task [3]. There are several ways of knowledge base formation methods [14]:

Manual - the knowledge is created by the real expert and knowledge engineer.

Semi-automatic - the knowledge is created partly by humans and partly in an automated way.

Fully-automatic - the knowledge is created without the participation of human experts.

In this paper we are analyzing automated knowledge base formation methods. One of such methods utilizes data import from specific domain ontologies [15]. It is a good way of knowledge base formation 
to use specific domain ontologies. This method is good because it allows us to reuse specific data, especially, where exact concepts are needed [18]. But the main disadvantage of this method is that there are several different ontologies languages, that means it is difficult to format knowledge base in universal way [8]. The other disadvantage is, that ontologies are not created every day, that means, we cannot get up to date data from ontologies format [17]. In the case of risk analysis, it is a must, since many threats, such as malware and vulnerabilities are dynamic in nature.

Another well known method for knowledge base formation is machine learning. Ethem Alpaydin (2010) suggested a simple method of machine learning for knowledge base formation [12]. There are several machine learning algorithms based on data classification (Bayes, Nearest neighbor method, regression, genetic algorithms, etc.) [16]. All of them are useful for prediction tasks where human can't make the prediction obviously. Machine learning algorithms don't need human interaction, they are self-learning systems and can work with various types of data. But the biggest disadvantage of such systems is that they are susceptible to errors [11]. It can take a lot time to discover the bug. Another limitation is related to the fact that the self-learning system need a lot of high-quality data [13]. It is not suitable for the information security analysis expert system, since, for example, the probability of a specific threat is a specific and dynamic value, and, as mention above, there is a need for actual up to date data.

The analysis of automated knowledge base formation methods for expert systems has shown that there are only a few such methods. The most popular are based on ontology integration and machine learning. The main disadvantage of ontology-based methods for risk analysis area is that data in ontology is typically static, outdated or suitable only for one risk assessment process part (e.g. for control selection), while machine learning is suitable when good datasets are available. It can be stated, that despite the fact that there are some automated methods for knowledge base formation, they are not suitable for creation of knowledge base of information security risk analysis expert system, responsible for the identification of threats in dynamic areas, such as malware and vulnerability threats.

\section{Integration of data on regional malware distribution from $\mathrm{Cy}$ - berthreat real-time map into the knowledge base}

This work presents an automated expert systems knowledge base formation method based on the acquisition of data from trusted and open websites providing current information on newly discovered threats, by reading their HTML code and extracting information between tags. HTML was chosen because it is widely used [4] and structured, so it can be easily transformed into another format.

The method proposed is shown on in Figure 1.

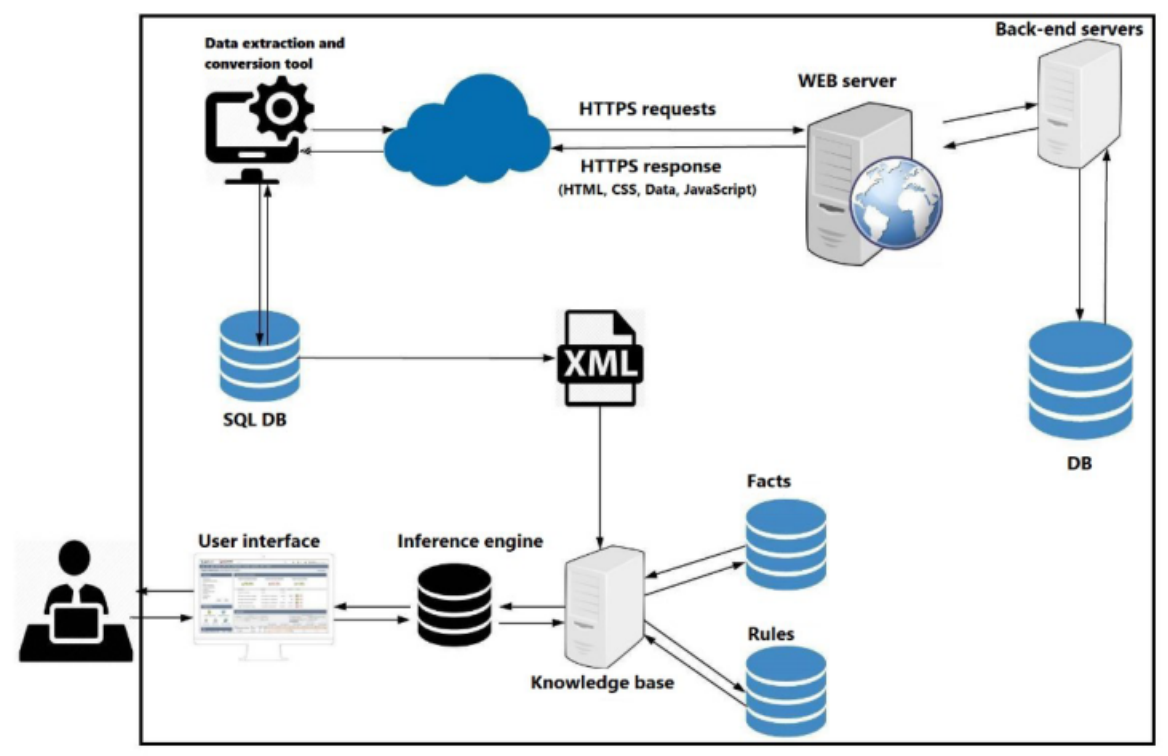

Figure 1: The architectural view of method implementation. 


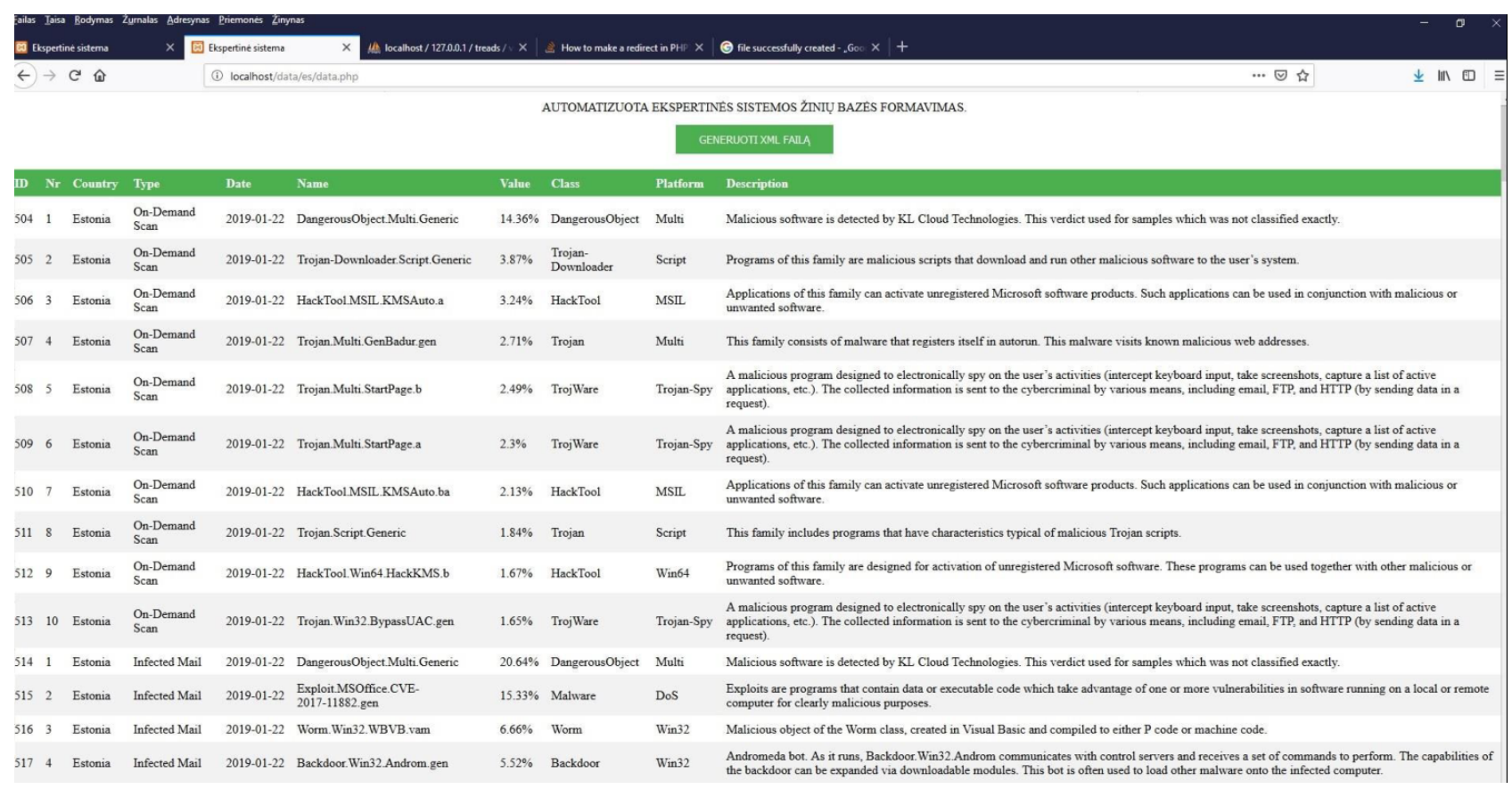

Figure 2: Example of automatically extracted data.

The proposed method has several steps:

1. Data extraction:

1.1. HTTPS requests to specified WEB page are sent.

1.2. HTTPS response is obtained from the WEB server.

1.3. Useful data about malware is extracted from HTML tags and stored to SQL DB.

2. Data conversion: all data in the SQL DB are converted to XML format.

3. Knowledge base formation: created XML file is imported to the existing expert system.

The method is implemented with a tool created in PHP language, which addresses the specified website by sending the HTTPS request. When a web server responds to the query, the tool reads the HTML code and processes the information between the specified tags. The collected data is stored in the SQL database. In this way, we get the needed knowledge from the web sites in an automated way, that can be later used in relevant risk calculations. Example of the automatically extracted data is shown in Figure 2.

The knowledge base formation is done by converting extracted data from SQL DB to XML format and importing it to JESS. Example of data conversion is shown in Figure 3.

The formed knowledge base was implemented on the Java expert system shell (JESS). All collected data was automatically imported into JESS using XML format. On Figure 4 an example of knowledge base import into JESS expert system prototype is demonstrated.

It is necessary to state, that automatically acquired data has formed only part of a knowledge base, i.e. was integrated into the existing knowledge base and was used only for estimation of malware probability used in risk calculation in the specific region. The prototype interacts with the user via a dialogue session. Risk identification is done by matching virus distribution platforms and platforms used in the organization. Also, the location of the organization and the prevalence of viruses in that area is evaluated. If an organization is operating in a country where the prevalence of the virus is recorded, the potential risk is calculated.

The proposed method was tested on data acquisition from the Cyberthreat real-time map website by Kaspersky Lab [19]. This site was chosen because it contains up to date information about known malicious code and its distribution location, in approximately 200 locations in total. In this work, information about 3 Baltic region countries was used: Lithuania, Estonia, Latvia. The extracted information contained the following information about malware: name, type, prevalence in the selected country, spreading platform, class, description, system, how it works, etc. Use case diagram of the method is shown in Figure 5. 


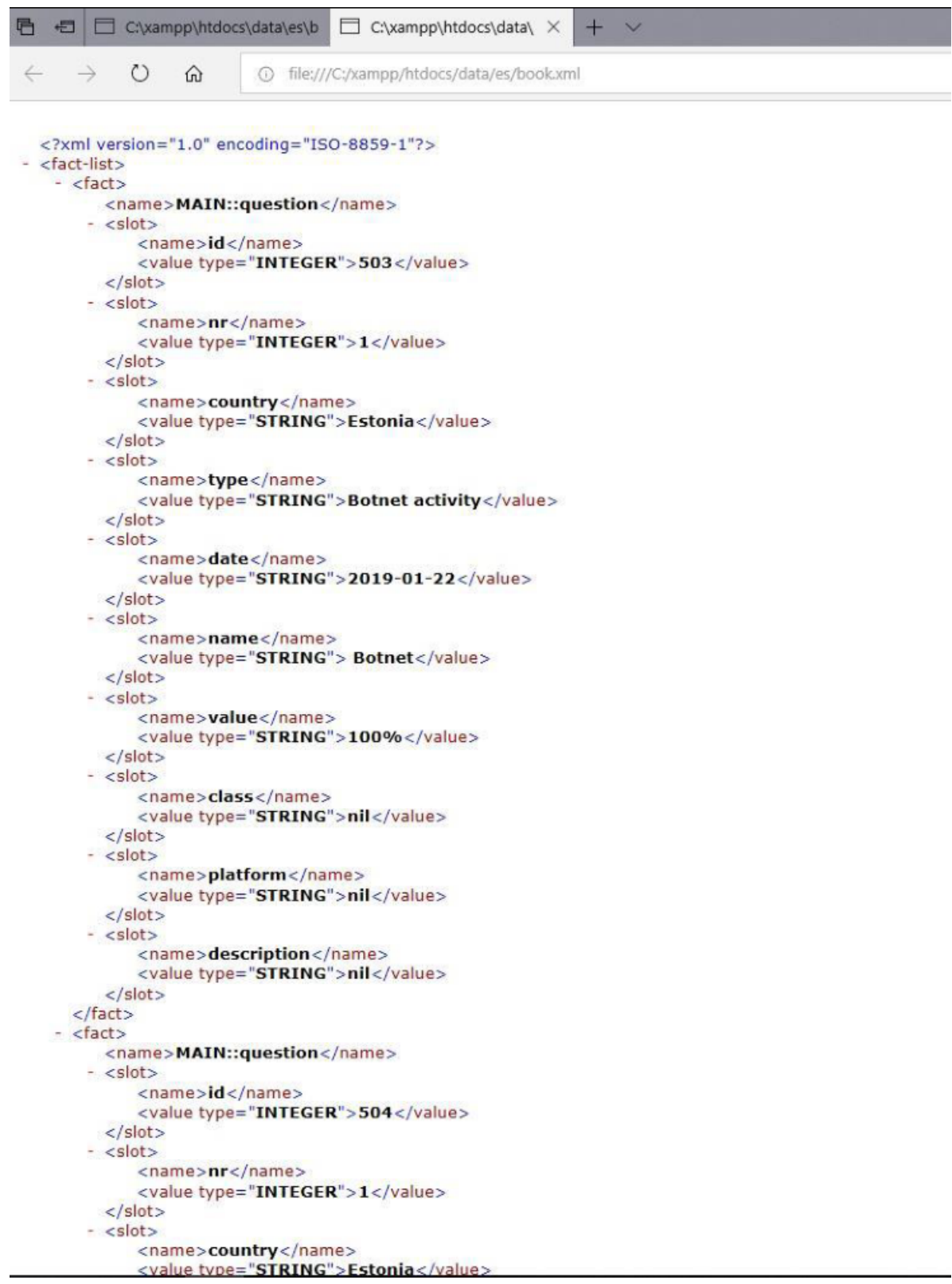

Figure 3: Example of data conversion to XML format.

While implementing the proposed method for the experiments, it was created a data extraction and conversion tool to get data from HTML, format the knowledge base and convert it to XML format. Also, a prototype of an expert system using a knowledge base with automatically acquired data was developed. The prototype was designed to perform the SME's information security risk assessment. 


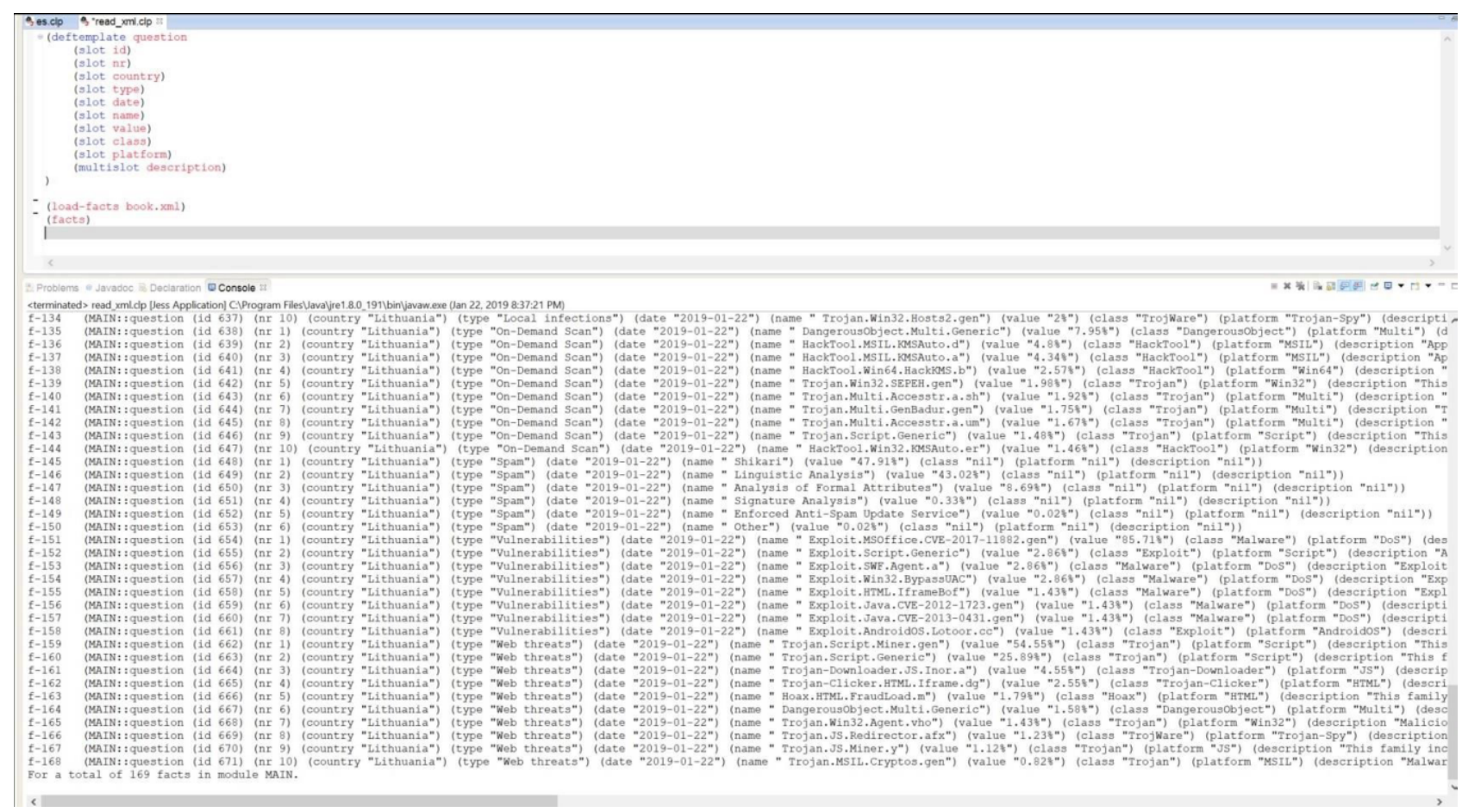

Figure 4: Example of the knowledge base import to the expert system.

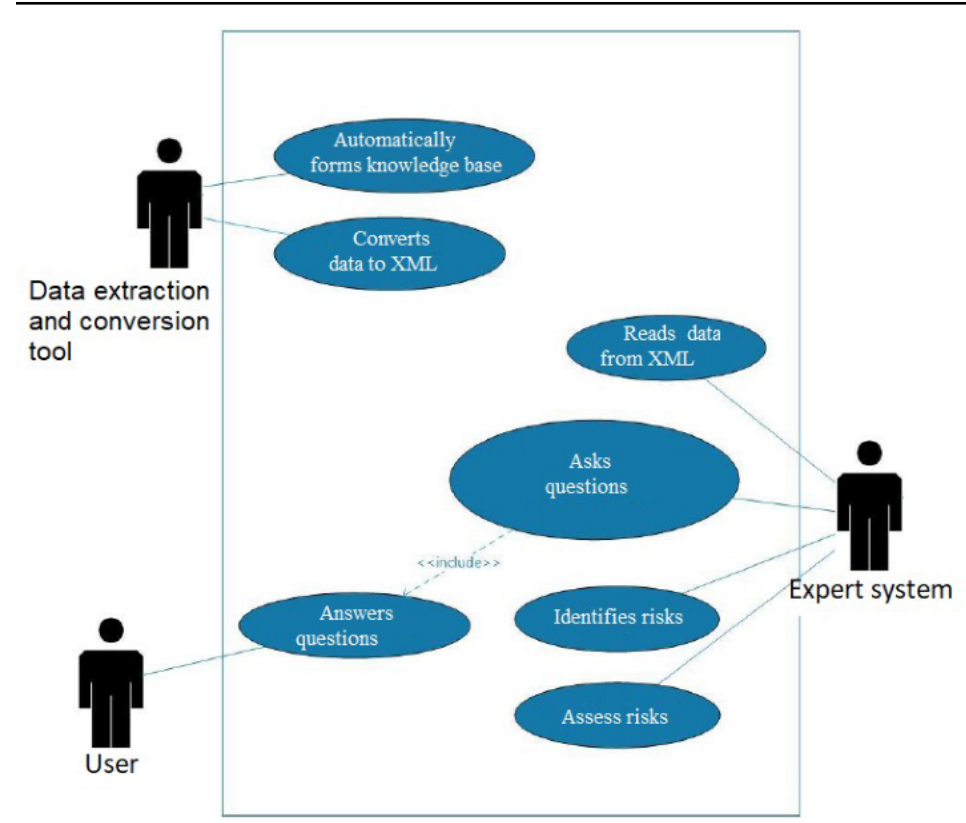

Figure 5: Method use case diagram.

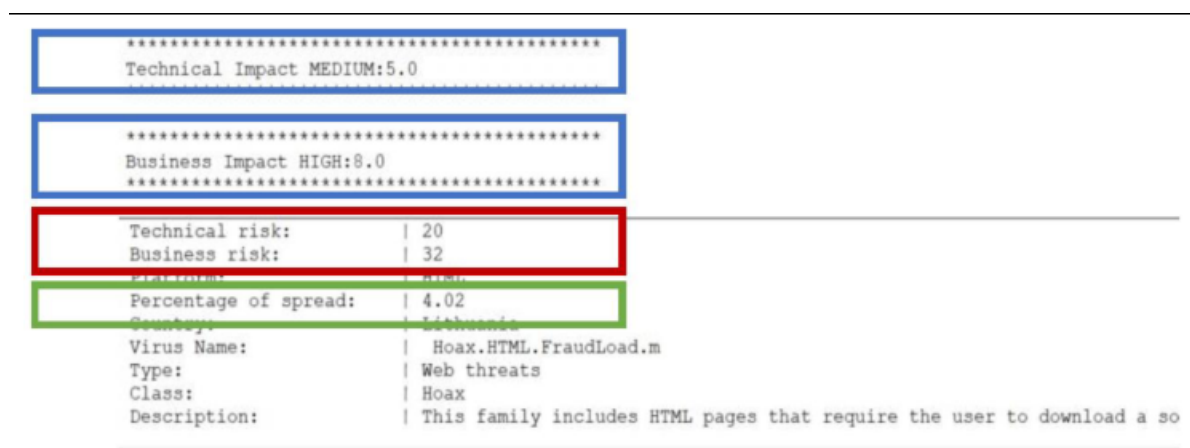

Figure 6: Example of the risk calculation. 


\section{Results and discussion}

During the experiment 225 web tags were scanned in total and 201 entries of knowledge base were formed automatically.

The proposed method has demonstrated a principal possibility of automated data extraction and import to the expert system knowledge base. Risk assessment, using automatically formed knowledge base, was conducted according to the OWASP methodology. According to the user answers, technical and business impacts were calculated in values from 0-9. The likelihood was evaluated according to the comparison of enterprise information systems platforms and malware platform.

Finally, the risk is calculated by formula: RISK $=$ LIKELIHOOD $*$ IMPACT. Example of the risk calculation is given in Figure 6.

Blue rectangles in the figure show impact, green - likelihood, red - the risk rate. The impact is evaluated by the business criteria, likelihood is a percentage of the malware spread in a specific region.

The main advantages of the method proposed are possibility to keep the knowledge base up to date with relevant information on current threats, to integrate information from multiple sources of information and to minimize workload while creating the knowledge base of an expert system. Limitations of the method are related to the changing structure of web sites providing data and the need to update the tool code manually because of that the further research is to be concentrated on tool adaptability for website structure.

\section{Conclusions}

The performed analysis has shown that increasing demand for automated information security risk assessment can be covered by the use of rules-based expert systems. However, the biggest problem in the development of this type of system is the formation and updating of the knowledge base especially in such a volatile area as cybersecurity. Manual methods of knowledge base formation are too slow, while existing semi-automatic and automatic knowledge base formation methods are concentrating on integration of static data, such as ontologies and can not depict the changing state in the cyberspace risk landscape. There is also a need to get risk assessment results fitted for the specific geographic location, since risk probabilities can be different in different regions due to variating malware population and other factors.

The new automated expert systems knowledge base formation method based on the acquisition of data from trusted and open websites providing current information on newly discovered malware and its geographic distribution was proposed. Method is based on scanning the HTML code, importing processed tag information into the database with further conversion into XML code and integration into the knowledge-base of a developed JESS-based expert system for geographically adapted malware risk assessment. Risk calculation is based on the widely accepted OWASP methodology. The method proposed differs from earlier methods by the ability to dynamically update the knowledge base rules and facts, thus reacting to the changing cyberthreat landscape, automatically present the emerging threats in a risk assessment with linking them to a geographic company location.

Kaspersky Lab's cyberthreat real-time map website was chosen as a data source for the method implementation tests. Data on geographical malware distribution was collected for 3 countries: Lithuania, Latvia and Estonia. The following data on cyber threats was collected in an automated way: malware description, related vulnerabilities, the prevalence of malware in the selected region, the malware spreading platform and typical impact on the system. The method tests on the developed JESS-based expert system has proved that automatically extracted data from the websites can improve the quality and expediency of risk assessment and provide geographically adapted results. 


\section{References}

[1] Agrawal, V.A. (2017). Comparative Study on Information Security Risk Analysis Methods, JCP, $12.1,57-67,2017$.

[2] Batista, L. O.; de Silva, G. A.; Araujo, V. S.; Araujo, V. J. S.; Rezende, T. S.; Guimaraes, A. J.; Souza, P. V. D. C. (2018). Fuzzy neural networks to create an expert system for detecting attacks by sql injection, The International Journal of Forensic Computer Science, 1, 8-21, 2018.

[3] Dheir, I.; Abu-Naser, S. S. (2019). Knowledge Based System for Diagnosing Guava Problems, International Journal of Academic Information Systems Research (IJAISR), 3(3), 9-15, 2019.

[4] Dua, S.; Du, X. (2016). Data mining and machine learning in cybersecurity, CRC press, 2016.

[5] Dzitac, I.; Barbat, B. E. (2009). Artificial intelligence + distributed systems = agents, International Journal of Computers Communications \& Control, 4(1), 17-26, 2009.

[6] Elsharif, A. A.; Abu-Naser, S. S. (2018). An Expert System for Diagnosing Sugarcane Diseases, International Journal of Academic Engineering Research (IJAER), 3(3), 19-27, 2019.

[7] Kireeva, N.; Pozdnyak, I.; Gazizulina, A. (2019). Filling a Knowledge Base for Expert System in Information Security, IOP Conference Series: Materials Science and Engineering, 618(1), 2019.

[8] Kless, D.; Milton, S.; Kazmierczak, E.; Lindenthal, J. (2015). Thesaurus and ontology structure: Formal and pragmatic differences and similarities, Journal of the Association for information science and technology, 66(7), 1348-1366, 2015.

[9] Li, D.; Cai, Z.; Deng, L.; Yao, X.; Wang, H. H. (2018). Information security model of block chain based on intrusion sensing in the IoT environment, Cluster Computing, 22(1), 451-468, 2019.

[10] Losonczi, P.; Necas, P.; Nad, N. (2016). Risk management in information security, Journal of Management, 28, 2016.

[11] Mohamed E. (2017). Comparative study of four supervised machine learning techniques for classifications, Information Journal of applied science and technology, 7(2), 5--18, 2017.

[12] Ramachandra, M. (2010). Information Mining, Web-Based Supply Chain Management and Digital Signal Processing: Methods for Effective Information Administration and Transmission, IGI Global, 223-231, 2010.

[13] Ristoski, P.; Paulheim, H. (2016). Web in data mining and knowledge discovery: A comprehensive survey, Journal of Web Semantics, 36, 1-22, 2016.

[14] Tandon, N.; Varde, A. S.; de Melo, G. (2017). Commonsense knowledge in machine intelligence, SIGMOD Record, 46(4), 2017.

[15] Vitkus, D.; Steckevicius, Z.; Goranin, N.; Kalibatiene, D.; Cenys, A. (2019). Automated Expert System Knowledge Base Development Method for Information Security Risk Analysis, International Journal of Computers Communications \& Control, 14(6), 743-758, 2019.

[16] Xiao, H.; Rasul, K.; Vollgraf, R. (2017). Fashion-MNIST: a novel image dataset for benchmarking machine learning algorithms, arXiv preprint, 2017.

[17] Yadav, U.; Narula, G. S.; Duhan, N.; Jain, V.; Murthy, B. K. (2015). Development and visualization of domain specific ontology using protege, Indian Journal of Science and Technology, 9(16), $1-7,2016$.

[18] Zhong, W.; Liu, S.; Wan, F.; Li, Z. (2018). Equipment selection knowledge base system for industrial styrene process, Chinese Journal of Chemical Engineering, 26(8), 1707-1712, 2018. 
[19] [Online]. Available: https://cybermap.kaspersky.com, Accesed on 27 March 2020.

[20] [Online]. Available: https://www.owasp.org/index.php/OWASP_Risk_Rating_Methodology, Accesed on 27 March 2020.

[21] [Online]. Available: https://jessrules.com/jess/download.shtml, Accesed on 27 March 2020.

\section{(c) (i) (8)}

Copyright (C)2020 by the authors. Licensee Agora University, Oradea, Romania.

This is an open access article distributed under the terms and conditions of the Creative Commons Attribution-NonCommercial 4.0 International License.

Journal's webpage: http://univagora.ro/jour/index.php/ijccc/

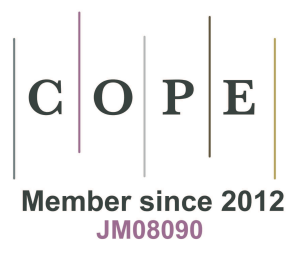

This journal is a member of, and subscribes to the principles of,

the Committee on Publication Ethics (COPE).

https://publicationethics.org/members/international-journal-computers-communications-and-control

Cite this paper as:

Vitkus, D.; Jezukeviciute, J.; Goranin, N. (2020). Dynamic Expert System-Based Geographically Adapted Malware Risk Evaluation Method, International Journal of Computers Communications \& Control, 15(3), 3865, 2020.

https://doi.org/10.15837/ijccc.2020.3.3865 